\title{
Microdroplet Operations in Polymeric Microtubes
}

\author{
Yu Zhang, Ziyun Wang, Declan New, and Michele Zagnoni* \\ Cite This: https://dx.doi.org/10.1021/acs.analchem.0c04360 \\ Read Online
}

ACCESS |

|lll Metrics \& More

Article Recommendations

Supporting Information

ABSTRACT: Microsystem technologies allow a plethora of operations to be achieved for microemulsion- and microdroplet-based assays, providing miniaturized, yet large-throughput capabilities to assist experimentation in analytical chemistry, biology, and synthetic biology. Many of such approaches have been implemented on-chip, using microfluidic and lab-on-a-chip technologies. However, the microfabrication of such devices relies on expensive equipment and time-consuming methods, thus hindering their uptake and use

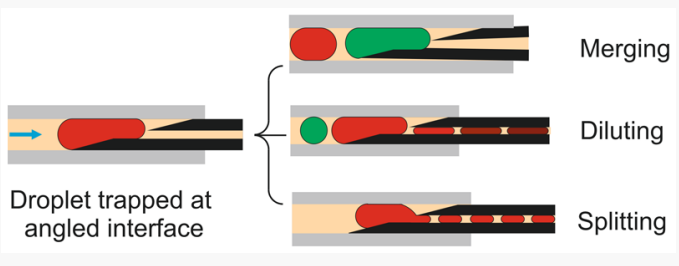
by many research laboratories where microfabrication expertise is not available.

Here, we demonstrate how fundamental water-in-oil microdroplet operations, such as droplet trapping, merging, diluting, and splitting, can be obtained using straightforward, inexpensive, and manually fabricated polymeric microtube modules. The modules are based on creating an angled tubing interface at the interconnection between two polymeric microtubes. We have characterized how the geometry and fluid dynamic conditions at this interface enabled different droplet operations to be achieved in a versatile and functional manner. We envisage this approach to be an alternative solution to expensive and laborious microfabrication protocols for droplet microfluidic applications.

\section{INTRODUCTION}

In recent years, droplet microfluidic technologies, ${ }^{1-3}$ where biological or chemical samples are compartmentalized within aqueous droplets surrounded by an oil phase, have been established as an effective, miniaturized, and high-throughput platform for analytical chemistry, ${ }^{4-6}$ synthetic biology, emulsification physics, ${ }^{10-12}$ and condition monitoring. ${ }^{13-15}$

Droplets with $\mathrm{pL}-\mathrm{nL}$ volumes can be passively generated by geometrical architectures ${ }^{16-19}$ or actively generated by valves ${ }^{20}$ and actuator-based platforms. ${ }^{21,22}$ Droplet operations, such as merging, $^{23}$ splitting, $^{24}$ and trapping, ${ }^{25,1,26}$ rely on specific geometrical features, which can be produced by advanced microfabrication techniques, such as soft lithography, ${ }^{27}$ threedimensional (3D) printing, ${ }^{28}$ laser cutting, ${ }^{29}$ micromachining, ${ }^{30}$ or injection molding. ${ }^{31,32}$ These microfabrication methods can be expensive, time-consuming, or demand microfabrication expertise. Although limited layouts of microfluidic modules are available commercially, the majority of these provide solutions for microdroplet generation rather than microdroplet operations. Therefore, droplet microfluidic devices are produced mainly in engineering research laboratories with a customizable format. ${ }^{33}$ These limitations make droplet microfluidics less accessible to the wider scientific communities that do not have extensive expertise in microsystem engineering. Therefore, simplified and inexpensive techniques that do not rely on microfabrication could enhance the uptake of droplet microfluidic technology and its use in many biological and chemical research laboratories.

Developing droplet microfluidic assays using commercially available tubing, , $^{34-37}$ capillaries, ${ }^{38-41}$ or connectors ${ }^{42,43}$ provides alternative solutions to chips-based microfluidics.
Utada and co-workers ${ }^{44}$ first used glass microcapillaries to form a coaxial geometry generating single and double emulsions. Chen et al. ${ }^{45}$ applied multiple glass capillaries to split single and double emulsions. The physical mechanisms underlying microdroplet generation using capillaries have been extensively studied, $^{46}$ and tubing-based microfluidics transporting and incubating microdroplets have been developed for cell-based assays, ${ }^{47,48}$ protein crystallizations, ${ }^{49}$ and synthesis of liposomes and nanomaterials. ${ }^{42,50-53}$ However, droplet operation modules based on tubing or capillaries are lagging behind, these still relying mainly on chip-based microfabricated layouts. Offering novel and simple approaches to achieve microdroplet operations using microtube-based microfluidics is therefore important to extend the assay capabilities to the wider scientific community.

In this paper, easy-to-replicate, manual protocols for assembling standard polymeric microtubes are presented that enable the most common microdroplet operations to be achieved. We describe how trapping, merging, diluting, and splitting of water-in-oil (W/O) microdroplets can be robustly obtained by interfacing manually prepared polymeric microtubes. We elucidate the physics that underpins droplet operations in microtubes and the way these can be exploited to develop advanced droplet protocols, discussing the

Received: October 15, 2020

Accepted: January 5, 2021 
functionality of the proposed methods and potential applications of such approaches.

\section{MATERIALS AND METHODS}

Materials. All chemicals were purchased from Sigma-Aldrich (U.K.) unless otherwise stated. Polyethylene (PE) tubing and polypropylene (PP) gel-loading pipette tips were purchased from Fisher Scientific (U.K.); poly(tetrafluoroethylene) (PTFE) tubing was purchased from Cole-Parmer (U.K.).

Experimental Setup to Investigate Droplets at Angled Interface. To facilitate the generation and fine-tuning of $\mathrm{W} / \mathrm{O}$ microdroplets, an in-house ${ }^{21,22}$ developed instrument was used to selectively aspirate water or oil fluid volumes into a loading microtube (PTFE, inner diameter $0.15 \mathrm{~mm}$, outer diameter 0.4 $\mathrm{mm}$ ) from a multi-well fluid container. Different aqueous solutions were placed in independent wells and covered by a common layer of hexadecane oil (Figure 1). The selection of the

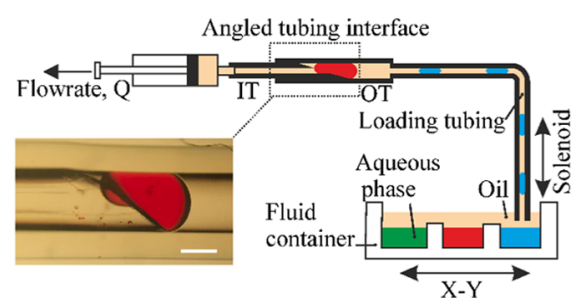

Figure 1. Experimental setup. W/O droplets were formed using an inhouse-developed instrument. A syringe pump continuously aspirated water or oil volumes into a loading tubing (inner diameter $0.15 \mathrm{~mm}$, outer diameter $0.4 \mathrm{~mm}$ ) from a fluid container, where different aqueous solutions were placed in wells and covered by a common oil layer. A computer-controlled $\mathrm{X}-\mathrm{Y}$ stage moved the container underneath the loading tube, so that the desired phase could be selected. Additionally, the loading tube was lowered in a time-controlled manner into the desired fluid, resulting in a $\mathrm{W} / \mathrm{O}$ droplet of defined volume to be formed. Droplets were subsequently transported to the angled tubing interface for the desired operation. Droplet operations at the angled interface were monitored under a microscope or recorded with a color camera for further analysis. Scale bar is $600 \mu \mathrm{m}$.

desired aqueous solution or oil phase was actuated by a solenoid, which transiently lowered the tip of the loading tube into the desired phase for the required time duration, generating $\mathrm{W} / \mathrm{O}$ droplets. Two stepper motors, controlled by in-house-developed $\mathrm{C}$-code via Arduino, actuated an $\mathrm{X}-\mathrm{Y}$ translation stage that determines the position of the wells with respect to the tubing tip. The aspiration was achieved using a $1 \mathrm{~mL}$ glass syringe (Duran, Germany) mounted on a syringe pump (AL-1000HP, World Precision Instrument) in withdrawal mode and set to a constant flow rate $(1-61.5 \mu \mathrm{L} / \mathrm{min})$. The size of droplets produced and tested in this work ranged from $\sim 10$ to $600 \mathrm{~nL}$. The generated droplets were subsequently transported from the loading tube to an angled tubing interface (dashed line rectangle in Figure 1), manually assembled by press-fitting microtubes, where microdroplet trapping, merging, diluting, and splitting operations were obtained.

Microtube Preparation and Manual Assembly. Polymeric tubing and/or the tapered, thin end of gel-loading pipette tips were used to create the desired tube-to-tube interface. Each interface consisted of an outside tube, "OT" (PE tube, inner diameter $0.58 \mathrm{~mm}$, outer diameter $0.96 \mathrm{~mm}$ ), and an inside tube, "IT" (PE tube, inner diameter $0.28 \mathrm{~mm}$, outer diameter 0.61 $\mathrm{mm}$, or PP-tapered gel-loading pipette tip, inner diameter 0.3 $\mathrm{mm}$, outer diameter $0.54 \mathrm{~mm}$ ). The IT was cut with a scalpel to create a desired angle with respect to the cross-sectional plane of the IT and then manually inserted in the OT, creating a leak-free, pressure-fit connection. The other extremity of the OT was then press-fit to the loading tube where droplets were generated. The other extremity of the IT was either connected to further tubing, interfaced to a collection vial (for sample connection), or collected into the syringe.

Imaging. An inverted microscope (Axiovert A1, Zeiss) was used for all experiments using objective lenses of $2.5 \times, 5 \times, 10 \times$, or 20X. Colored images/videos were acquired through a color camera connected to the microscope eyepiece. Fluorescent images were acquired using an EMCCD LucaR camera (Andor Technologies) via Andor Solis software, using a fluorescein isothiocyanate (FITC) filter. The intensity of fluorescent droplets were calculated by averaging the intensity values of a region of interest of 100 pixels in the center of the droplet. ImageJ software (v1.46r) was used to analyze and process recorded videos.

\section{RESULTS}

The interconnection created between the $\mathrm{OT}$ and IT provides the key element that determines the implementation of basic droplet operations, such as merging, dilution, and splitting. These are dependent on the size of the droplets, their velocity, and the geometry of the tubing interface.

Microdroplet Trapping Mechanism. All microdroplet operations in this paper are based on the fabrication of an "angled" OT-IT interface, where W/O droplets are trapped. To this end, polymeric microtubes were chosen to guarantee the desired phase wetting properties that promoted $\mathrm{W} / \mathrm{O}$ emulsions. The OT was obtained from a microtube with a circular cross section, while two types of microtubes were used for the IT, either a tapered microtube (thin end of gel-loading pipette tip) with a circular cross section or a microtube with a flattened tip to obtain an elliptical cross section. The tip of the IT was cut to a certain angle $(\alpha)$ with respect to the plane of its cross section and manually inserted into the OT, as schematically shown in Figure 2A. The protocol used for obtaining a tube with an elliptical cross section is illustrated in Figure S1. The distance that the IT was inserted inside the OT was not critical, as long as the tubes created a leak-free junction.

A W/O droplet is trapped at the angled interface when a balance is reached between the Laplace pressure forces around the droplet and hydrodynamic forces that push the droplet toward the interface. Three steps (Figure 2B and Movie S1) describe the droplet trapping sequence: (i) a droplet approaches the interface with a constant velocity according to the flow rate applied, Q. (ii) The frontend of the droplet is deformed at the angled interface, reducing its radius of curvature with respect to its backend, which remains in the OT (point a in Figure 2B) and remains almost unchanged. The droplet moves forward until an oil bypass is created. (iii) The droplet stops and remains trapped when an equilibrium between Laplace pressure forces and hydrodynamic forces is reached. Ultimately, any condition that pushes the droplet frontend to travel further into the angled tubing interface and completely obstructs its opening (point $b$ in Figure 2B) will force the droplet to flow into the IT.

The trapping condition is described by eq 1 , where $P_{\mathrm{a}}$ is the pressure at the backend of the droplet, $P_{\mathrm{b}}$ is the pressure at the frontend of the droplet (with $P_{\mathrm{a}}-P_{\mathrm{b}}=Q \times R$, where $Q$ is the average flow rate determined by the withdrawal pump and $R$ is the average hydraulic resistance of the bypass gap), $r_{\mathrm{b} 1}$ and $r_{\mathrm{b} 2}$ are the radii of curvature of the frontend of the droplet when 


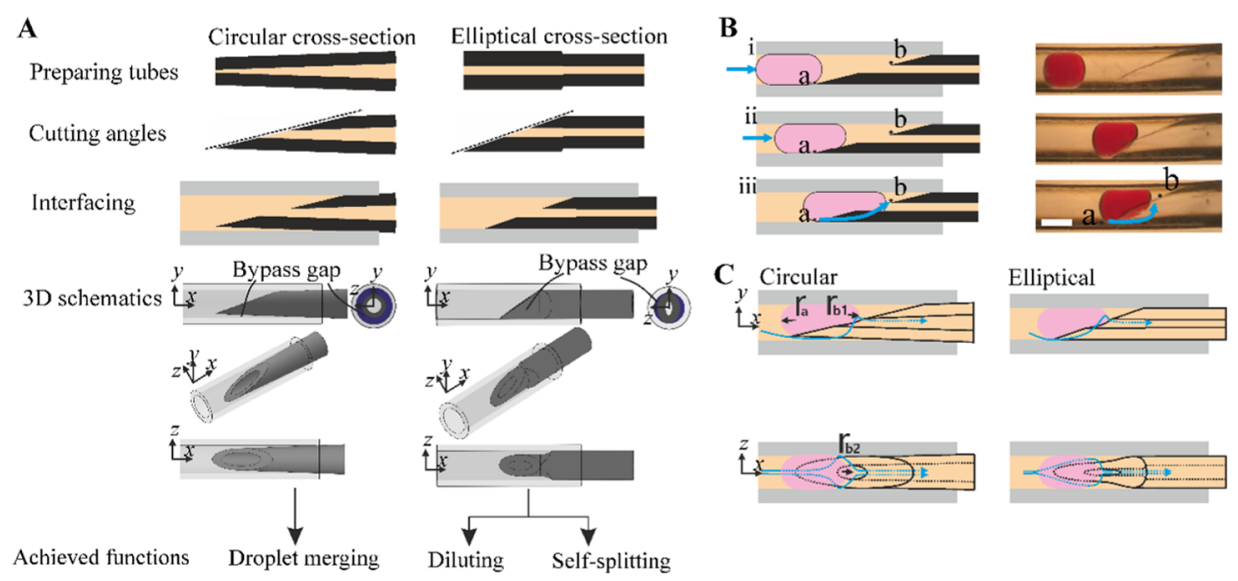

Figure 2. Droplet trapping at an angled OT-IT interface. (A) $2 \mathrm{D}$ and 3D schematics showing the fabrication of angled tubing interface: the IT is made of either a tapered microtube with circular cross section (thin end of a gel-loading pipette tip) or a flattened tube with an elliptical cross section. The IT is cut to the desired angle and inserted into the OT. (B) Side view schematic (left column) and images (right column) of the three key steps to achieve droplet trapping at the angled interface (circular cross section or elliptical cross section). First, the droplet moves toward the interface with a constant velocity (i). When the droplet approaches the angled interface (ii), the frontend of the droplet is guided onto the sloped IT, while its backend remains in the OT. The droplet continues to move forward until a bypass gap for the oil phase (from point a to point b) is created into the IT. When a balance is reached between the Laplace pressure and the hydrodynamic pressure around the droplet, this eventually stops and remains trapped (iii). Scale bar is $600 \mu \mathrm{m}$. (C) Schematic representation of the side view and top view of a trapped droplet at the angled interface for both types of IT. The blue lines show different trajectories of the oil flow path from OT to IT around the droplet.

trapped at the angled interface, and $r_{\mathrm{a}}$ is the approximated radius of curvature of the backend of the droplet.

$$
P_{\mathrm{a}}-P_{\mathrm{b}} \leq \gamma\left(\frac{1}{r_{\mathrm{b} 1}}+\frac{1}{r_{\mathrm{b} 2}}-\frac{2}{r_{\mathrm{a}}}\right)
$$

As $r_{\mathrm{b} 1}$ and $r_{\mathrm{b} 2}$ are always smaller than $r_{\mathrm{a}}$, the leading droplet interface is "more rigid" than the backend, creating a redistribution of the droplet surface, which allows the oil to flow from the OT to the IT via the bypass gap. Only higher values of hydrodynamic pressure difference will be able to move the frontend of the droplet further into the IT (detailed derivation is shown in the Supporting Information). This trapping mechanism is similar to that obtained by chip-based microfluidic layouts in the literature based on bypass channels $^{23,54,55}$ or surface energy traps. ${ }^{56,57}$ The trapping mechanism can also be explained considering the interfacial or surface free energy of the $\mathrm{W} / \mathrm{O}$ droplet. A droplet has the tendency to minimize its surface energy by reducing its surface area, $E=\gamma \times S$ ( $S$ is the surface area of the droplet at a given time, $\gamma$ is the interfacial tension). ${ }^{57}$ During trapping, the droplet's surface gradually increases while entering the angled interface. The droplet is trapped when the droplet surface free energy equalizes that created by the hydrodynamic forces on the droplet.

An effective way to fine-tune the trapping condition defined by eq 1 is to modify the geometrical shape of the IT by altering its circular cross section into an almost elliptical cross section by flattening the tubing extremity (Figure S1). This procedure significantly decreases one radius of curvature of the IT and consequently increases the Laplace pressure at the frontend of the trapped droplet. Concurrently, it also induces a larger bypass gap to be obtained as more space is created between the IT and the OT (3D schematics in Figure 2A), which ultimately leads to a smaller hydraulic resistance $R$. A further parameter affecting the trapping mechanism is the slope of the angled interface, $\alpha$ (Figure 3A). By decreasing the angle $\alpha$, the length of the angled interface increases $\left(D_{\mathrm{OT}} \times \cot (\alpha)\right)$, thus enabling larger droplets to be trapped.

To study the droplet trapping efficiency of differently sloped angled interfaces, we arbitrarily selected three different values of $\alpha$, approximately $5^{\circ}, 20^{\circ}$, and $45^{\circ}$. For each angle, we characterized experimentally the relationship between the largest volume of the droplet that could be trapped at the interface with respect to the flow rate magnitude applied with the syringe pump, for both circular and elliptical cross sections of the IT. To compare the system performance in different experimental conditions, we measured the largest droplet volumes that could be trapped at an angled interface and normalized these values to the theoretical volume given by ( $1 /$ $12+\cot \alpha / 8) \times \pi \times D_{\mathrm{OT}^{3}}{ }^{3}$ (Figure 3A). Lines in Figure 3B,C show the normalized maximum volumes of droplets trapped at the angle interface in the cases of circular and elliptical IT cross sections, respectively. Regions positioned below the lines represent droplets that could be trapped at the corresponding angled interface, while those above the lines could not be trapped. The results show that angled interfaces with elliptical cross sections present stronger trapping ability for the same flow rate than those of circular cross sections. When plotting the same data of Figure 3B,C as absolute droplet volume values (Figure S2), the results show that smaller values of the angle $\alpha$ are able to trap larger droplet volumes. In our experimental setup, droplets with diameter smaller than $\sim 0.3 \mathrm{~mm}$ were not considered, as these were smaller than the IT diameter.

Microdroplet Merging. Controllable pairwise droplet merging ${ }^{23,58}$ is a fundamental process in many applications to trigger chemical reactions ${ }^{42,59}$ for DNA sequencing ${ }^{60,61}$ or protein crystallization. ${ }^{62}$ The proposed angled interface enables pairwise (Movie S2) or multiple droplet merging (Movie S3) to be obtained (Figure 4). This operation was obtained using an angled interface composed of an IT with a circular cross section.

Five steps underpin this operation (Figure 4A): (i) while one droplet (green) is trapped at the angled interface, a second droplet (red) approaches the first one; (ii) coalescence of the two droplets is achieved upon contact as no surfactants are used. 

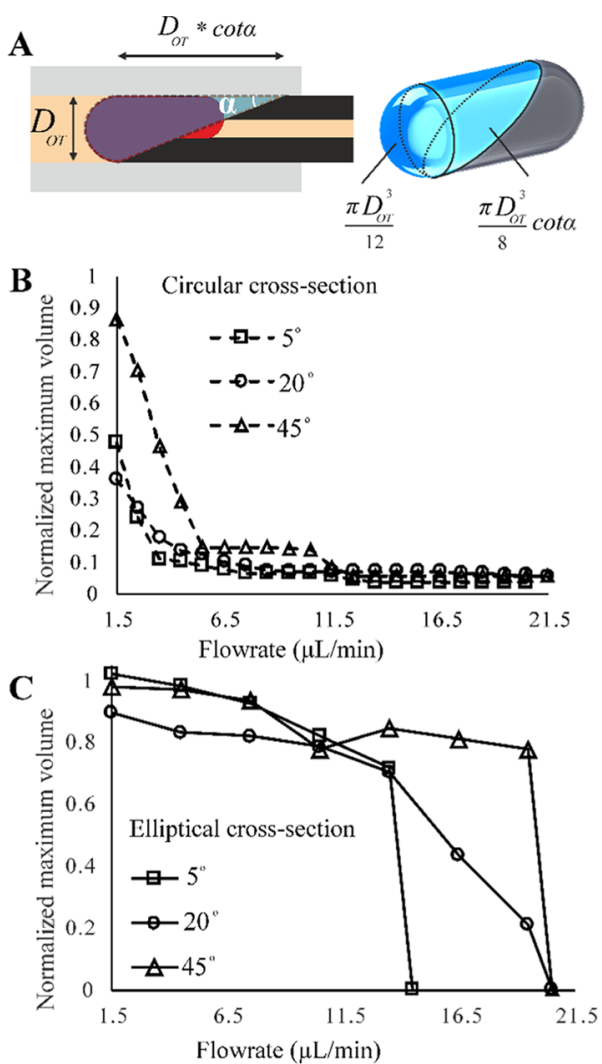

Figure 3. Condition to trap droplets at angled tubing interfaces. (A) Schematic illustrating the geometrical configuration of the maximum droplet volume that can be housed at the angled tubing interface according to the parameter $\alpha$ (derivation in the Supporting Information). (B, C) Plots showing the trapping efficiency of the angled tubing interface for three angles, $\alpha \sim 5^{\circ}, \sim 20^{\circ}$, and $\sim 45^{\circ}$, with respect to the same range of flow rates. The largest volume of the trapped droplets was normalized to the theoretical value of a trapped droplet at the angled interface, given by $(1 / 12+\cot \alpha / 8) \times \pi \times D_{\mathrm{OT}}{ }^{3}$. Regions under the curve represent conditions that enable droplet trapping.

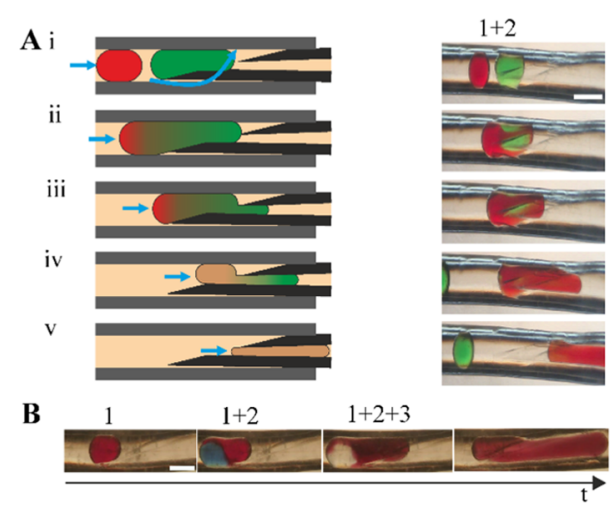

Figure 4. An angled interface enables controlled droplet merging operations. (A) Schematic diagrams and snapshots that depict the key processes of droplet trapping, merging, and release. (i) The first droplet (green) is trapped, waiting for a second droplet (red) to reach the interface. The blue arrow indicates the flow direction. (ii) Two droplets are merged. (iii) The merged droplet moves toward the IT. (iv, v) The merged droplet travels into the IT and is released from the trap without breaking. (B) A representative example of the merging of three droplets (red, blue, and white). Scale bars are $600 \mu \mathrm{m}$.
Once coalescence is initiated, (iii) the droplet starts moving into the IT. Subsequently, (iv) the resulting droplet enters the IT and (v) is released from the angled interface without breakup. An essential condition for this operation to take place is that, following droplet merging, the resulting droplet has a normalized volume greater than 1 (point above the curve in Figure 3B), when the equilibrium of forces (eq 1) can no longer be maintained. The same principle applies when merging multiple droplets (Figure 4B and Movie S3). Therefore, the volume of each droplet must be carefully controlled during generation and in relation to the angle $\alpha$, as their cumulative volume at the angled interface determines how many droplets can be merged. The smaller the droplet size, the higher the number of droplets that can be merged for a given angled interface.

For a constant flow rate, the parameter that determines the merging rate is the time lag between any two generated droplets. This was controlled by appropriately selecting the droplet generation frequency using our experimental setup. In the two examples shown (Figure 4A,B), the merging rate is $\sim 0.14 \mathrm{~Hz}$ for pairwise droplet merging and $\sim 0.05 \mathrm{~Hz}$ for triple droplet merging.

Microdroplet Dilution. The formation of serial dilutions is an important operation in many chemical and biological assays. A droplet-based microfluidic dilutor ${ }^{55,63}$ constitutes a powerful chemistry tool for high-throughput screening. However, this function typically requires sophisticated designs and microfabricated layouts. The angled tubing interface enables precise droplet assay dilution (Movie S4).

To achieve a reproducible serial dilution process, ${ }^{55,63,64}$ the volume of the diluent solution added into the mother sample is normally designated to be identical to the diluted volume extracted from the mother sample (Figure 5A). The elliptical angled interface facilitates the size of the diluent droplet to match that of the diluted droplet. This implies that the size of the mother droplet (trapped at the angled tubing interface) remains constant, i.e., the trapped droplet is "metered". ${ }^{55,64}$ Figure 5B$\mathrm{D}$ demonstrates that the angled interface with an elliptical cross section is capable of precisely metering aqueous droplets.

There are six steps to complete one dilution process (Figure $5 B$ ). First, a mother droplet (red) with a volume $V_{\mathrm{m}}$ and concentration $C_{0}$ is trapped at the angled interface (i) until a diluent droplet (green), with volume $V_{\mathrm{d}}$, merges with the former. After merging (ii), the total volume of the merged droplet $\left(V_{\mathrm{m}}+\right.$ $V_{\mathrm{d}}$ ) is greater than the corresponding maximum volume allowed for trapping a droplet at the angle interface and it fully blocks the oil bypass gap. As a result, the frontend of the merged droplet is pushed into the IT (iii). Immediately after merging, the diluent volume is confined to the backend of the droplet, while the frontend is flowing into the IT. After a short period of time (iv), the volume of the droplet in the OT returns to its original value and the oil bypass gap is re-established. This results in a Rayleigh-Plateau instability at the angled interface (white dot circle in Figure 5A). Consequently (v), a droplet with the same volume of the diluent droplet is pinched off, leaving a mother droplet restored to its original volume $V_{\mathrm{m}}$ and a concentration $C_{1}$, where $C_{1}<C_{0} \times\left(1-V_{\mathrm{d}} / V_{\mathrm{m}}\right)$. During the time elapsed between (iii) and (v), the diluent does not have enough time to propagate and diffuse to the droplet frontend. By appropriately spacing diluent droplets apart, the viscous shear forces induced by the bypass flow on the droplet $\mathrm{W} / \mathrm{O}$ interface ensure thorough mixing (vi) in the mother droplet (i.e., $\sim 40 \mathrm{~s}$ for the 

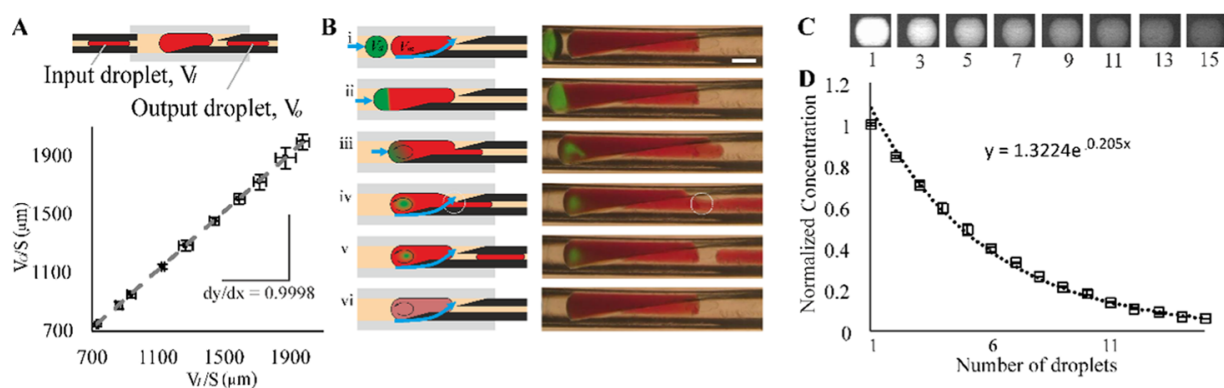

Figure 5. An angled interface enables droplet dilutor operations. (A) Size of the input droplet (diluent) is identical with that of the output droplet (diluted) ( $n=5)$. (B) Key steps of a droplet dilutor: (i) a mother droplet (red) is trapped at the angled interface waiting for diluent droplets (green) to coalesce. (ii) After a diluent droplet is merged with the mother droplet, the bypass gap is blocked. (iii) The frontend of the merged droplet is pushed into the IT. (iv) The merged droplet reduces its volume in the OT and Rayleigh-Plateau instability emerges around the neck in the IT. (v) The front tip of the merged droplet pinches off (v), leaving the mother droplet at the angled interface. Relying on the viscous shear induced by the bypass flow, the trapped mother droplet is thoroughly mixed after time (vi). The blue arrows indicate the direction of oil flow, while the black arrows indicate the inner droplet eddies. (C, D) A representative example of 15 droplets pinched off from the mother droplet containing originally calcein. The fluorescent intensity of each droplet is normalized to the value of the first droplet pinched off. Experiments were repeated three times. Scale bars are $600 \mu \mathrm{m}$.

flow rates tested in this work), due to inner droplet eddies. This last aspect determines the precision of the dilutor.

To demonstrate the capability of the angled interface dilutor, a mother droplet, containing $100 \mu \mathrm{M}$ calcein, was diluted 15 times (Figure 5C,D) with blank droplets. By setting the period of incoming diluent droplets to $\sim 1 \mathrm{~min}$, thorough mixing was achieved throughout the process. Fluorescent measurements of the 15 released droplets, emerging from the mother droplet, follow an excellent exponential fit (Figure 5D).

Microdroplet Splitting. Fine-tuning the size of microdroplets and creating monodispersed populations are common requirements in many droplet-based assays. ${ }^{65}$ A convenient way of achieving this is to split a long plug into many smaller and equally sized droplets using a Y-junction. ${ }^{66}$ This function can be obtained in the proposed setup using an angled interface composed of an IT with an elliptical cross section (Movie S5).

To achieve this, a droplet is generated and transported to the angled interface using a flow rate higher than its critical trapping value (Figure 3C). In this condition, a balance between surface tension forces and hydrodynamic forces is never achieved and this can be described as

$$
P_{\mathrm{a}}-P_{\mathrm{b}}>\gamma\left(\frac{1}{r_{\mathrm{b} 1}}+\frac{1}{r_{\mathrm{b} 2}}-\frac{2}{r_{\mathrm{a}}}\right)
$$

This condition results in the frontend of the droplet being always pushed into the IT by hydrodynamic pressure. The elliptical cross section creates small radii of curvature when the droplet interface enters the IT, which, combined with interface instabilities, produces the periodic formation of an intermittent oil bypass gap inducing droplet pinch-off, similar to step (iv) in Figure 5A. The process of pushing the frontend of a droplet into IT with subsequent pinch-off is repeated until the droplet at the angled interface is depleted (Figure 6A). The process of continuously pushing and pinching off a droplet is similar to that obtained in passive microfluidic devices. ${ }^{66}$

The size of droplets that can be split is equal or smaller than the theoretical value given by $(1 / 12+\cot \alpha / 8) \times \pi \times D_{\mathrm{OT}^{3}}{ }^{3}$. This depends on the inner and outer tubing diameters and on the angle $\alpha$. Larger tubing diameters and smaller values of $\alpha$ allow trapping of larger droplets. For a given droplet volume, the size of the daughter droplets depends on the magnitude of the flow rate, where higher flow rates produce smaller droplets with higher monodispersity. As an example, the polydispersity of

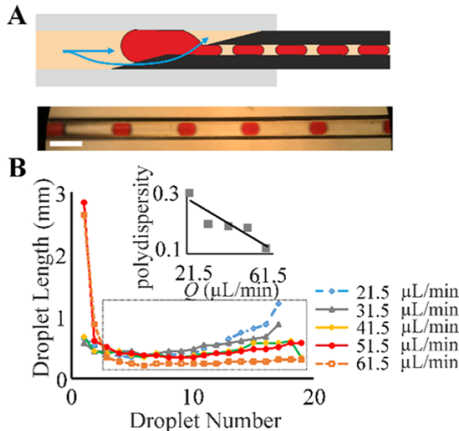

Figure 6. An angled interface enables the splitting of plugs into daughter droplets. (A) Schematic of a plug splitting into smaller daughter droplets and image of a train of daughter droplets. (B) Characterization of size polydispersity of daughter droplets under different flow rates. When calculating polydispersity, the first two droplets were excluded. Typically, a plug of $0.6 \mu \mathrm{L}$ could be split into $\sim 20$ droplets. Higher flow rate enables smaller polydispersity of sizes of droplets. Each condition was repeated at least three times. Scale bar is $600 \mu \mathrm{m}$.

droplet sizes (excluding the first two droplets) decreased from 30 to $10 \%$ for increasing flow rates from 21.5 to $61.5 \mu \mathrm{L} / \mathrm{min}$ (Figure 6B), identifying an expected trend where higher flow rates produced more monodisperse droplet populations.

\section{DISCUSSION}

The proposed tubing modules and associated methodologies are the first examples of microdroplet operations that do not rely on chip-based microfabricated structures.

We have presented a manually constructed, easy-to-assemble microtube system for droplet microfluidic circuit operations without the need for microfabrication expertise, laborious protocols, or expensive microfabrication equipment. The use of polymeric microtubes has several advantages over other microfluidic formats. First, polymeric microtubes, especially PE, PP, or PTFE tubing, are flexible, economic, and commercially available with a large range of dimensions, thus making this method highly accessible to different scientific communities. Second, the variety of tubing materials have intrinsic surface properties to fine-tune the type of emulsions $(\mathrm{W} / \mathrm{O}$ or $\mathrm{O} / \mathrm{W})$ and oil phases (e.g., hydrocarbon or fluorinated oils) that can be used. Third, this method is highly modular, as the different 
angled tubing interfaces presented here can be serially extended or connected in parallel, relying just on manual pressure-fitting protocols. This approach is also amenable to other tubing microfluidic modules ${ }^{44,67}$ and, being based on nonporous and non-gas-permeable materials, offers advantages in minimizing evaporation of the phases, a common problem in elastomerbased (i.e., poly(dimethylsiloxane), PDMS) microfluidic devices.

The factors that affect the droplet trapping at an angled tubing interface are the size of the droplets, the flow rate, the angle $\alpha$, and the geometry of the tubing cross section. The ratio of the inner to outer tubing affects the droplet trapping condition, the operation, and the size of the resulting droplets, directly influencing the geometrical parameters of the droplets at the angled interface (Figure S2). These geometrical parameters can be compensated by appropriately selecting the flow rate. Moreover, the viscosity of the oil phase is directly proportional to the hydraulic resistance of the tubing and, consequently, affects the trapping capability at the angled interface (eqs 1 and S7). For increasing values of dynamic viscosity, an increasing hydraulic resistance is obtained and, therefore, decreasing values of the flow rate or larger cross sections of the inner tubing are required to maintain the pressure difference constant. When the droplet volume is larger than a critical value (Figure 3), a droplet cannot be trapped, as an oil bypass flow cannot be obtained and an equilibrium between surface tension and flow forces cannot be achieved. When this occurs at a circular angled interface, the droplet is released from the interface typically without breakup. This feature was exploited to achieve droplet trap-mergerelease mechanisms. Differently, at elliptical angled interfaces, droplet breakup is easier to achieve due to the geometrical cross section inducing higher values of localized pressure at the interface. This feature was exploited to achieve droplet metering and droplet dilution operation working across the critical droplet value, while non-equilibrium conditions allowed droplet splitting.

Surfactants are commonly used in microdroplet operations to prevent coalescence or interdroplet exchange of encapsulated substances. In our system, the use of surfactants did not affect the functionality of trapping or splitting operations, but that of droplet merging and diluting, as surfactants prevent the coalescence of droplets that occur during such operations. In the future, the possible application of external electrical fields ${ }^{56}$ could overcome these limitations. Furthermore, we expect that the use of surfactants with the angled interface modules can yield to other droplet-based applications. ${ }^{54,68}$

Overall, the main advantage of the proposed tubing-based approach is the flexibility in replacing chip-based microsystems with inexpensive and disposable tubing modules. However, a trade-off exists between decreasing the complexity of the system and its performance with respect to device-based droplet microfluidics.

In our experimental setup, the flow rate was controlled by a syringe pump in withdrawal mode, an instrument widely accessible in many biochemistry labs, while microdroplets were formed in a low-throughput manner using an in-house-built robotic system (also commercially available). The latter enabled the automated generation of droplets with the desired size and composition. The proposed microtube system operates at smaller rates (10's Hz), larger droplet volumes ( $\mathrm{nL}$ instead of $\mathrm{pL}$ ), and therefore smaller droplet throughput than chip-based platforms. However, other tubing-based droplet protocols ${ }^{42,43}$ can be used to obtain higher-throughput applications. Addi- tionally, while a syringe pump (or equivalent instrumentation) may be used for flow control, other options have been reported in the literature to generate droplets, such as gravity-fed systems. ${ }^{69,70}$ If combined with equipment-free flow actuation methods, tube-based droplet techniques can be deployed for medium-throughput and miniaturized assays in remote or resource-poor areas. Finally, polymeric microtubes are deformable, a useful feature for the development of wearable or textile devices. $^{71}$ The angled interface module can also be used as a droplet meter to precisely dispense liquid solutions. For example, the serial combination of several meters and dilutor tubing modules can reproduce chip-based configurations to dilute samples without the need for droplet generation, as shown for microfluidic domino layouts. ${ }^{64}$

\section{CONCLUSIONS}

Polymeric tubes with sub-millimeter bore dimensions are low cost, available in hydrophilic and hydrophobic materials, and widely used in biochemistry labs. Here, we have shown experimental protocols to achieve specific microdroplet-based functions using manual protocols for the fabrication of polymeric microtube setups. Capabilities of droplet splitting, merging, and diluting tubing modules were characterized, highlighting the advantages that such easy-to-use setup can provide. Therefore, we expect this work to facilitate the uptake and development of cost-effective, chip-free microdroplet-based assays in research laboratories that do not have access to microfabrication facilities, lack in microsystem development expertise, or would like an opportunity to decrease fabrication costs.

\section{ASSOCIATED CONTENT}

\section{(3) Supporting Information}

The Supporting Information is available free of charge at https://pubs.acs.org/doi/10.1021/acs.analchem.0c04360.

Equation derivation, illustration and methodology for elliptical tubing interface fabrication, and data on droplet trapping conditions (PDF)

An example of a $\mathrm{W} / \mathrm{O}$ droplet trapped at the angled interface (Movie S1) (MP4)

Two W/O droplets merging at an angled interface with a circular cross section (Movie S2) (MP4)

Three W/O droplets merging at an angled interface with a circular cross section (Movie S3) (MP4)

Serial dilution of $\mathrm{W} / \mathrm{O}$ droplets at an angled interface with an elliptical cross section (Movie S4) (MP4)

Splitting of a W/O droplet at an angled interface with an elliptical cross section (Movie S5) (MP4)

\section{AUTHOR INFORMATION}

\section{Corresponding Author}

Michele Zagnoni - Centre for Microsystems and Photonics, EEE Department, University of Strathclyde, Glasgow G1

1XW, U.K.; (1) orcid.org/0000-0003-3198-9491;

Email: michele.zagnoni@strath.ac.uk

\section{Authors}

Yu Zhang - Centre for Microsystems and Photonics, EEE Department, University of Strathclyde, Glasgow G1 1XW, U.K. 
Ziyun Wang - Centre for Microsystems and Photonics, EEE Department, University of Strathclyde, Glasgow G1 1XW, U.K.

Declan New - Centre for Microsystems and Photonics, EEE Department, University of Strathclyde, Glasgow G1 1XW, U.K.

Complete contact information is available at:

https://pubs.acs.org/10.1021/acs.analchem.0c04360

\section{Notes}

The authors declare no competing financial interest.

\section{ACKNOWLEDGMENTS}

This work was supported by EPSRC (EP/N031849/1).

\section{REFERENCES}

(1) Seemann, R.; Brinkmann, M.; Pfohl, T.; Herminghaus, S. Rep. Prog. Phys. 2012, 75, No. 016601.

(2) Guo, M. T.; Rotem, A.; Heyman, J. A.; Weitz, D. A. Lab Chip 2012, $12,2146-2155$.

(3) Teh, S. Y.; Lin, R.; Hung, L. H.; Lee, A. P. Lab Chip 2008, 8, 198220.

(4) Zheng, B.; Roach, L. S.; Ismagilov, R. F. J. Am. Chem. Soc. 2003, $125,11170-11171$.

(5) Du, W. B.; Li, L.; Nichols, K. P.; Ismagilov, R. F. Lab Chip 2009, 9, 2286-2292.

(6) Guzowski, J.; Gizynski, K.; Gorecki, J.; Garstecki, P. Lab Chip 2016, 16, 764-772.

(7) Brouzes, E.; Medkova, M.; Savenelli, N.; Marran, D.; Twardowski, M.; Hutchison, J. B.; et al. Proc. Natl. Acad. Sci. U.S.A. 2009, 106, 14195-14200.

(8) Cole, R. H.; Tang, S. Y.; Siltanen, C. A.; Shahi, P.; Zhang, J. Q.; Poust, S.; et al. Proc. Natl. Acad. Sci. U.S.A. 2017, 114, 8728-8733.

(9) Schlicht, B.; Zagnoni, M. Sci. Rep. 2015, 5, No. 9951.

(10) Bremond, N.; Thiam, A. R.; Bibette, J. Phys. Rev. Lett. 2008, 100, No. 024501.

(11) Holtze, C.; Rowat, A. C.; Agresti, J. J.; Hutchison, J. B.; Angile, F. E.; Schmitz, C. H. J.; et al. Lab Chip 2008, 8, 1632-1639.

(12) Zagnoni, M.; Baroud, C. N.; Cooper, J. M. Phys. Rev. E 2009, 80, No. 046303.

(13) Chen, D.; Du, W. B.; Liu, Y.; Liu, W. S.; Kuznetsov, A.; Mendez, F. E.; et al. Proc. Natl. Acad. Sci. U.S.A. 2008, 105, 16843-16848.

(14) Wang, M.; Roman, G. T.; Schultz, K.; Jennings, C.; Kennedy, R. T. Anal. Chem. 2008, 80, 5607-5615.

(15) Nightingale, A. M.; Leong, C. L.; Burnish, R. A.; Hassan, S. U.; Zhang, Y.; Clough, G. F.; et al. Nat. Commun. 2019, 10, No. 2741.

(16) Thorsen, T.; Roberts, R. W.; Arnold, F. H.; Quake, S. R. Phys. Rev. Lett. 2001, 86, No. 4163.

(17) Anna, S. L.; Bontoux, N.; Stone, H. A. Appl. Phys. Lett. 2003, 82, 364-366.

(18) Cramer, C.; Fischer, P.; Windhab, E. J. Chem. Eng. Sci. 2004, 59, 3045-3058.

(19) Priest, C.; Herminghaus, S.; Seemann, R. Appl. Phys. Lett. 2006, 88, No. 024106.

(20) Churski, K.; Michalski, J.; Garstecki, P. Lab Chip 2010, 10, 512518.

(21) Du, W. B.; Sun, M.; Gu, S. Q.; Zhu, Y.; Fang, Q. Anal. Chem. 2010, 82, 9941-9947.

(22) Gielen, F.; van Vliet, L.; Koprowski, B. T.; Devenish, S. R.; Fischlechner, M.; Edel, J. B.; et al. Anal. Chem. 2013, 85, 4761-4769.

(23) Niu, X.; Gulati, S.; Edel, J. B.; deMello, A. J. Lab Chip 2008, 8, 1837-1841.

(24) Link, D. R.; Anna, S. L.; Weitz, D. A.; Stone, H. A. Phys. Rev. Lett. 2004, 92, No. 054503.

(25) Tan, W. H.; Takeuchi, S. Proc. Natl. Acad. Sci. U.S.A. 2007, 104, 1146-1151.
(26) Casadevall i Solvas, X.; deMello, A. Chem. Commun. 2011, 47, 1936-1942.

(27) Unger, M. A.; Chou, H. P.; Thorsen, T.; Scherer, A.; Quake, S. R. Science 2000, 288, 113-116.

(28) Bhargava, K. C.; Thompson, B.; Malmstadt, N. Proc. Natl. Acad. Sci. U.S.A. 2014, 111, 15013-15018.

(29) Klank, H.; Kutter, J. P.; Geschke, O. Lab Chip 2002, 2, 242-246.

(30) Guckenberger, D. J.; de Groot, T. E.; Wan, A. M. D.; Beebe, D. J.; Young, E. W. K. Lab Chip 2015, 15, 2364-2378.

(31) Owens, C. E.; Hart, A. J. Lab Chip 2018, 18, 890-901.

(32) Becker, H.; Gartner, C. Anal. Bioanal. Chem. 2008, 390, 89-111.

(33) Ding, Y.; Howes, P. D.; deMello, A. J. Anal. Chem. 2020, 92, $132-149$.

(34) Asano, S.; Takahashi, Y.; Maki, T.; Muranaka, Y.; Cherkasov, N.; Mae, K. Sci. Rep. 2020, 10, No. 7685.

(35) Kerr, C. B.; Epps, R. W.; Abolhasani, M. Lab Chip 2019, 19, $2107-2113$

(36) Bandulasena, M. V.; Vladisavljevic, G. T.; Benyahia, B. J. Colloid Interface Sci. 2019, 542, 23-32.

(37) Wu, P.; Wang, Y.; Luo, Z. F.; Li, Y. T.; Li, M. F.; He, L. Q. Lab Chip 2014, 14, 795-798.

(38) Li, Y. K.; Wang, K.; Xu, J. H.; Luo, G. S. Chem. Eng. J. 2016, 293, 182.

(39) Zhu, P.; Kong, T.; Kang, Z.; Tian, X.; Wang, L. Sci. Rep. 2015, 5, No. 11102 .

(40) Meng, Z. J.; Wang, W.; Liang, X.; Zheng, W. C.; Deng, N. N.; Xie, R.; et al. Lab Chip 2015, 15, 1869-1878.

(41) Wu, P.; Wang, Y.; Luo, Z.; Li, Y.; Li, M.; He, L. Lab Chip 2014, 14, 795-798.

(42) Nightingale, A. M.; Phillips, T. W.; Bannock, J. H.; de Mello, J. C. Nat. Commun. 2014, 5, No. 3777.

(43) Benson, B. R.; Stone, H. A.; Prud'homme, R. K. Lab Chip 2013, $13,4507-4511$.

(44) Utada, A. S.; Lorenceau, E.; Link, D. R.; Kaplan, P. D.; Stone, H. A.; Weitz, D. A. Science 2005, 308, 537-541.

(45) Chen, Y.; Gao, W.; Zhang, C.; Zhao, Y. Lab Chip 2016, 16, $1332-1339$.

(46) Guerrero, J.; Chang, Y. W.; Fragkopoulos, A. A.; FernandezNieves, A. Small 2020, 16, No. 1904344.

(47) Siltanen, C. A.; Cole, R. H.; Poust, S.; Chao, L.; Tyerman, J.; Kaufmann-Malaga, B.; et al. Sci. Rep. 2018, 8, No. 7913.

(48) Jiang, C. Y.; Dong, L.; Zhao, J. K.; Hu, X.; Shen, C.; Qiao, Y.; et al. Appl. Environ. Microbiol. 2016, 82, 2210-2218.

(49) Zheng, B.; Tice, J. D.; Roach, L. S.; Ismagilov, R. F. Angew. Chem., Int. Ed. 2004, 43, 2508-2511.

(50) Bottaro, E.; Nastruzzi, C. Mater. Sci. Eng., C 2016, 64, 29-33.

(51) Bezinge, L.; Maceiczyk, R. M.; Lignos, I.; Kovalenko, M. V.; deMello, A. J. ACS Appl. Mater. Interfaces 2018, 10, 18869-18878.

(52) Lignos, I.; Morad, V.; Shynkarenko, Y.; Bernasconi, C.; Maceiczyk, R. M.; Protesescu, L.; et al. ACS Nano 2018, 12, 55045517

(53) Sui, J.; Yan, J.; Liu, D.; Wang, K.; Luo, G. Small 2020, 16, No. 1902828

(54) Zagnoni, M.; Cooper, J. M. Lab Chip 2010, 10, 3069-3073.

(55) Niu, X.; Gielen, F.; Edel, J. B.; deMello, A. J. Nat. Chem. 2011, 3, 437-442.

(56) Fradet, E.; McDougall, C.; Abbyad, P.; Dangla, R.; McGloin, D.; Baroud, C. N. Lab Chip 2011, 11, 4228-4234.

(57) Dangla, R.; Lee, S.; Baroud, C. N. Phys. Rev. Lett. 2011, 107, No. 124501 .

(58) Niu, X. Z.; Gielen, F.; deMello, A. J.; Edel, J. B. Anal. Chem. 2009, $81,7321-7325$.

(59) Song, H.; Chen, D. L.; Ismagilov, R. F. Angew. Chem., Int. Ed. 2006, 45, 7336-7356.

(60) Hosokawa, M.; Nishikawa, Y.; Kogawa, M.; Takeyama, H. Sci. Rep. 2017, 7, No. 5199.

(61) Lan, F.; Demaree, B.; Ahmed, N.; Abate, A. R. Nat. Biotechnol. 2017, 35, 640-646. 
(62) Zheng, B.; Ismagilov, R. F. Angew. Chem., Int. Ed. 2005, 44, 2520-2523.

(63) Postek, W.; Kaminski, T. S.; Garstecki, P. Analyst 2017, 142, 2901-2911.

(64) Korczyk, P. M.; Derzsi, L.; Jakiela, S.; Garstecki, P. Lab Chip 2013, 13, 4096-4102.

(65) Chen, D.; Du, W.; Liu, Y.; Liu, W.; Kuznetsov, A.; Mendez, F. E.; et al. Proc. Natl. Acad. Sci. U.S.A. 2008, 105, 16843-16848.

(66) Postek, W.; Kaminski, T. S.; Garstecki, P. Lab Chip 2017, 17, 1323-1331.

(67) Chen, Y. P.; Gao, W.; Zhang, C. B.; Zhao, Y. J. Lab Chip 2016, 16, $1332-1339$.

(68) Zhang, Y.; Bracken, H.; Woolhead, C.; Zagnoni, M. Biosens. Bioelectron. 2020, 150, No. 111920.

(69) Moon, B. U.; Abbasi, N.; Jones, S. G.; Hwang, D. K.; Tsai, S. S. Anal. Chem. 2016, 88, 3982-3989.

(70) van Steijn, V.; Korczyk, P. M.; Derzsi, L.; Abate, A. R.; Weitz, D. A.; Garstecki, P. Biomicrofluidics 2013, 7, No. 024108.

(71) Xi, W.; Kong, F.; Yeo, J. C.; Yu, L.; Sonam, S.; Dao, M.; et al. Proc. Natl. Acad. Sci. U.S.A. 2017, 114, 10590-10595. 